\title{
Analysis of Gate-waiting Delays at Major US Airports
}

\author{
Jianfeng Wang; John F. Shortleł Juan Wang $\$$ Lance Sherry ${ }^{\S}$ \\ Center for Air Transportation Systems Research, George Mason University, Fairfax, VA, 22030, USA
}

\begin{abstract}
Airport gates are one of the congestion points of the air transportation system. When an arriving flight lands on a runway, it is possible that it cannot pull into its gate. We define this phenomenon as gate-waiting delay. This paper analyzes the degree to which gate waiting is a problem and the functional causes of gate waiting. Analysis of flight performance data for the OEP 35 airports $^{\mathrm{a}}$ for the summer of 2007 identified that: (i) Significant gate-waiting delays, in which more than $30 \%$ of arriving aircraft are delayed, occurred at 10 of the OEP 35 airports, (ii) major gate-waiting delays are rare events (e.g., once a month at ATL), (iii) Gate-waiting delays are usually different among major carriers due to different scheduling strategies. For example, Delta schedules more aggressively on their gate capacity (overscheduling) than JetBlue at JFK. (iv) Functional origins of gate-waiting delay included compressed arrivals, extended gate occupancy times, reduced number of gates, and inflexible queueing disciplines (across carriers and within one carrier). Many of these origins are related to schedule disruptions which are the main common factor identified in the worst days. The methodology for analysis, the results, and the implications of these results are discussed.
\end{abstract}

\section{Introduction}

The objective of this paper is to evaluate the degree to which airport gates are a limiting resource in the flow of airplanes arriving to and departing from an airport. As an airplane arrives to and departs from an airport, it passes through several potential choke points. These include runways, taxiways, ramps, gates, and so forth. Depending on the demand and number of resources, different points in this process may become constrained and act as choke points.

The runways (and associated constraints) are usually the limiting resource in the flow through the airport. This is because various separation requirements limit the maximum number of operations that can be safely handled in a given time interval. Once an airplane has landed and has taxied to its gate, it can often pull directly into the gate. But if the gate is unavailable, the arriving aircraft may have to wait. If the expected waiting time is long, the aircraft may be sent to a "penalty box" until a suitable gate becomes available (Figure 1).

While gates are not usually thought of as a choke point, this paper aims to evaluate the extent to which this can be the case. Conditions under which gates act as a limiting resource may increase over time if capacity is added to other parts of the system (say, through increased capabilities proposed in NextGen) without an appropriate analysis of the matching gate capacity.

At a high level, we can view this problem from the perspective of queueing theory. ${ }^{2}$ Delays are fundamentally related to one of several issues: (a) higher scheduled demand than capacity or over-scheduling (b) a larger arrival rate than scheduled, (c) a lower service rate than scheduled, or (d) a smaller number of servers than scheduled. In the context of this paper, the gates are the servers and the service rate is the rate that aircraft can be turned at the gate. For example, if an aircraft has a longer turnaround time than scheduled, this is effectively a reduction in the service rate of the gate. For a queueing system operating near capacity,

${ }^{*}$ PhD student, Systems Engineering and Operations Research Department; Research Assistant, Center for Air Transportation Systems Research (CATSR), member

${ }^{\dagger}$ Associate Professor, Systems Engineering and Operations Research Department Department, non-member

${ }^{\ddagger}$ Master student, Statistics Department; Research Assistant, CATSR, non-member

$\S$ Executive Director, CATSR; Associate Professor, Research, Systems Engineering and Operations Research Department, member

${ }^{\mathrm{a}}$ commercial U.S. airports with significant activity ${ }^{1}$ 


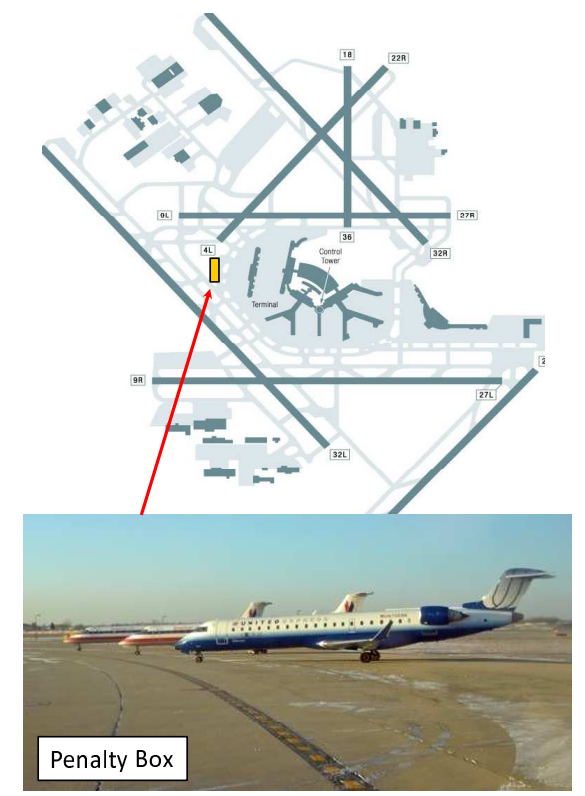

Figure 1. Penalty box at Chicago O'Hare airport

reducing the service rate - even by a little bit - can lead to significant delays. ${ }^{2}$ In summary, gate-related delays are attributable in some way to either (a) over-scheduling (b) too many arriving aircraft, (c) long turnaround times, or (d) reduced usable gates. A second goal of this paper is to identify functional origins of gate-related delays related to these fundamental issues.

This paper is organized as follows. Section II summarizes the literature related to gate delay. Sections III and IV discuss several methods for estimating gate delay using the BTS ${ }^{\mathrm{b}}$ and $\mathrm{ASPM}^{\mathrm{c}}$ databases. Section V presents analysis of gate-waiting delay at the OEP 35 airports. It also presents a closer analysis of several extreme days at ATL.

The results indicate that on most days, gate delays do not significantly limit throughput of the airport. However, on certain bad days, the limited number of gates leads to extreme delays. The problematic days appear to be linked to disruptions in the schedule. Disruptions in the schedule have the tendency of keeping aircraft on the ground longer, effectively reducing the gate "service rate". For example, a disruption in the schedule may require using a different crew on a given flight. In a similar manner, if a flight is cancelled, then the aircraft may either be held at the gate or reassigned to a later flight, thus increasing the time at the gate. Ground delay programs for outbound flights may also increase the number of gates required as airlines choose not to enplane or not to push back these flights. Finally, disruptions in the schedule may lead to sets of aircraft arriving at the same time.

\section{Background and Related Literature}

\section{A. Effects of Gate-waiting Delay}

Gate waiting affects airline operations in many ways. First, gate-waiting delay increases the cost of operating a flight, including fuel burn, crew cost, aircraft life and engine life. Gate waiting can also affect surface operations if airports do not have enough space to hold aircraft waiting for a gate. For example, BOS does not have holding pads where aircraft can wait for gates, so held aircraft may contribute to surface congestion. ${ }^{3}$

Gate-waiting delay usually leads to arrival delay. Arrival delay may have a propagation effect on an aircraft's next departure and on other aircraft's departure due to crew and passenger connections. If the airport is the passenger's final destination, the passenger trip delay is equal to this flight's arrival delay. But if gate-waiting delay causes passengers to miss their connections, the passenger trip delay will be much

\footnotetext{
${ }^{\mathrm{b}}$ Bureau of Transportation Statistics

${ }^{\mathrm{c}}$ Aviation System Performance Metrics
} 
higher than the gate-waiting delay. ${ }^{4}$

An airline's schedule is limited by gate availability. Gate shortage can decrease schedule volume that can otherwise be achieved. This has been an issue, for example, at ATL. Since building the international E concourse prior to the 1996 Olympics, no new gates had been added until 2007, when three new gates were added. In a 2007 earnings call, AirTran Holdings Inc. officials said that the company took quarterly losses at the end of 2006 in order to "control" the gates and add new flights, ${ }^{5}$ which means that AirTran had to lease the gate in 2006 so that they could have access to it in 2007.

\section{B. Gate-waiting Delay Reasons}

Over-scheduling can be a reason for gate-waiting delay. For example, "observations at Logan Airport showed that despite the limited gate capacity, some airlines overschedule their gates and consequently have simultaneously more aircraft on the ground than the number of available gates." ${ }^{6}$ But, in general, gate capacity is not overscheduled. "Airlines normally schedule gates for only up to $80 \%$ occupancy, knowing that they will need extra facilities to cope with random occurrences." 7

High aircraft arrival rates and long gate occupancy times (compared with scheduled operations) can also be a reason for gate-waiting delay. "A 'gate occupied' delay may occur because an arrival aircraft is early; however, it is often caused by departure delays from leaving the gate on schedule, due to gate operations or due to other constraints such as absorbing ground delays." 6

It is also possible that an aircraft does not go to a gate even if the gate is available (carrier and aircraft type match). For example, based on communication with a gate manager of United Airlines at O'Hare airport, United Airlines usually let an arriving aircraft wait for its original assigned gate even if there is an open alternative, when the gate conflict is detected late. The reason is that they do not want to redirect crew and passengers on the ground to another gate with a short lead time unless the expected gate-waiting delay is too long. Finally, the number of usable gates may be reduced in inclement weather or when ramp staff are unavailable. ${ }^{8}$

\section{Mitigation}

Airlines can mitigate gate-waiting delays by decreasing schedule volume and implementing more efficient gate assignment algorithms. Gate assignment is usually handled in three phases. ${ }^{9}$ The first planning phase occurs several months before the day of operation. Ground controllers check that a feasible gate assignment can be made with the proposed flight schedule. The second phase involves the development of a single-day plan prior to the start of the actual day of operation. The third phase revises the daily plan throughout the day of operation due to irregular operations such as delays, bad weather, mechanical failures and maintenance requirements. ${ }^{10}$

Previous research on gate assignments has stressed improving the performance of initial gate assignments. The problem has been modeled as an integer program, ${ }^{11,12}$ a mixed integer program ${ }^{10,13}$ and a network flow problem. ${ }^{14,15}$ Recently, some models have been developed to focus on gate changes. ${ }^{16}$ Some research has even evaluated the robustness of the initial gate assignment plan and the real-time gate changes necessary to meet the stochastic flight delays that occur in real operations. ${ }^{15,17,18}$ Such research has typically considered small stochastic disruptions in the schedule and not major disruptions. This paper shows that major schedule disruptions can have a significant impact on gate congestion, and corresponding gate assignment strategies need to be developed for these scenarios.

Airports can mitigate gate-waiting delays by reserving more common gates, encouraging gate usage exchange among carriers, and using hard stands. "The greater the proportion of common and exclusive-use gates the greater the reduction in inefficiency ${ }^{\mathrm{d}}$. Common use gates means the airport controls gate use and will ensure they are used most efficiently. Similarly when a gate has been given to a carrier for exclusive use it is in the carriers best interest to use the resource efficiently. As the proportion of preferential-use gates rises, efficiency falls. A carrier which has preferential treatment may try to use gates as a barrier to entry and not necessarily use them efficiently." 19

\footnotetext{
${ }^{\mathrm{d}}$ Terminal efficiency was defined as number of passengers and pounds of cargo served given number of runways, number of gates and other airport resources.
} 


\section{Modeling of the Taxi-in and Turnaround Processes}

Queueing models and integer programming models have been used to model the taxi-in process. ${ }^{3,20,21}$ The minimum service time under ideal turnaround operations has also been modeled. ${ }^{22}$ But the impact of schedule disruptions on turnaround operations has not been studied thoroughly. Modeling turnaround times in irregular operations could lead to a better understanding of gate utilization.

More accurate departure demand prediction is another potential benefic of improved ground-operations modeling. Departure demand is based on the push-back time, which is determined by gate arrival time, the turnaround time, and the schedule. Departure demand prediction is important to air traffic controllers for runway and taxiway scheduling. But it has been found that the prediction of departure demand is not accurate because the turnaround time can not be predicted well. ${ }^{6}$ Also, a gate shortage can result in pushing aircraft back from the gate, even though the downstream departure runway is a constraint. ${ }^{6}$ This has implications on matching the runway and gate capacity under disruption scenarios. If departure runway capacity drops while arrival runway capacity remains the same, gate demand is higher because holding at the gate is preferred.

\section{Data Sources}

Gate-waiting delay is not recorded in actual operations and thus is not directly available in any database. In particular, although several databases record the taxi-in time of individual flights, they do not break this time into its component parts, such as the delay specifically due to waiting for a gate. Thus, gate-waiting delay must be inferred or approximated using other information available. This section discusses several data sources - in particular, the BTS and ASPM databases - and associated fields that can be used to infer or approximate gate-waiting delay. The precise algorithms used to estimate gate-waiting delay are discussed in Section IV.

\section{A. BTS Airline On-Time Performance Database}

This database is maintained by BTS, part of DOT ${ }^{\mathrm{e}}$. This database has only one table, On-Time Performance. This table contains on-time arrival data for non-stop domestic flights by major air carriers and provides such additional items as departure and arrival delays, origin and destination airports, flight numbers, scheduled and actual departure and arrival times, cancelled or diverted flights, taxi-out and taxi-in times, air times, and non-stop distances. ${ }^{23}$ Key fields in this table are flight date, destination airport, carrier, scheduled arrival time, "wheels-on" time (the time that an aircraft lands on the runway, $\mathrm{see}^{24}$ for a precise definition), "gate-in" time (the time that an aircraft pulls into a gate), "gate-out" time (the time that an aircraft pushes back from a gate), and "taxi-in" time (the difference between "wheels-on" time and "gate-in" time).

\section{B. ASPM Database}

The ASPM database is maintained by the FAA ${ }^{\mathrm{f}}$. Two tables used in this paper are the "taxi time" table and the "individual flight" table. Key fields are the "wheels-on" time (the time that an aircraft lands on the runway), the "gate-in" time (the time that an aircraft pulls into a gate), the actual taxi-in time, the taxi-in delay, and the unimpeded taxi-in time. Other key fields are airport, carrier code, and season. The actual taxi-in time is the gate-in time minus the wheels-on time. The taxi-in delay is actual taxi-in time minus the unimpeded taxi-in time. The unimpeded taxi-in time is an estimated value using a regression model. ${ }^{25}$ It represents the time it would take for an aircraft to taxi in the absence of delay-causing factors such as congestion or weather. ${ }^{26}$ The unimpeded taxi-in time is broken down by calendar year, by season, by carrier, and by airport (for all carriers and airports reporting in the ASQP and Aeronautical Radio, Incorporated [ARINC] data). In 2001, there were 14 reporting carriers and about 300 reporting airports.

\section{Comparison of BTS and ASPM Database}

Key differences between the ASPM and BTS databases are the following ${ }^{23,26}$ (see also Table 1):

\footnotetext{
e United States Department of Transportation

${ }^{\mathrm{f}}$ Federal Aviation Administration
} 
- BTS includes data for air carriers that have at least one percent of total domestic scheduled-service passenger revenues (20 carriers); ASPM includes data for all carriers.

- BTS includes data for operations to and from airports that account for at least one percent of the nation's total domestic scheduled-service passenger enplanements (32 airports); ASPM includes data for 77 airports.

- BTS includes cancelled flights; ASPM does not.

- BTS does not include international flights; ASPM does. (BTS covers nonstop scheduled-service flights between points within the United States, including territories.)

- BTS does not include information regarding aircraft type; ASPM does.

- BTS contains the tail number of flights; ASPM only contains the tail number of flights that are also in BTS.

Table 1. Coverage of BTS and ASPM databases

\begin{tabular}{l|c|c}
\hline \hline & BTS & ASPM \\
\hline Carrier & 20 US major & All \\
Airport & 32 US major & 77 \\
Cancelled flights & Yes & No \\
International flights & No & Yes \\
Aircraft type & No & Yes \\
Tail number & Yes & BTS Flights Only \\
\hline \hline
\end{tabular}

Table 2 shows the number of flights recorded in the data sources for two example days at two airports. In both days, ASPM records more flights than BTS, due to the inclusion of international flights. Also, the gap between ASPM and BTS is larger for JFK than for ATL, since JFK has a higher percentage of international flights. As a reference, the third line gives the average number of daily flights for each airport (averaged over one year, 2007) obtained from Airport Council International. The last line shows the number of flights observed from the public website www.flightstats.com.

Table 2. Flights recorded in various data sources

\begin{tabular}{l|cc}
\hline \hline & ATL & JFK \\
Source & $6 / 5 / 07$ & $7 / 3 / 07$ \\
\hline BTS & 2239 & 675 \\
ASPM & 2690 & 1175 \\
Average Daily Flights (ACI) & 2724 & 1223 \\
Flightstats.com & 2794 & 1324 \\
\hline \hline
\end{tabular}

\section{Other Data Sources}

To get the number of gates and their users, this paper uses information from each airport's website, as well as the public website www.flightstats.com. This site provides flight information by airport and day, including the flight's final gate assignment, flight number, arrival time, departure time, carrier, and aircraft type. For example, we found that 179 different gates were used at ATL on June 11, 2007. We use this value as an estimate of the number of gates at ATL in the summer of 2007. This paper also uses a Sensis Aerobahn TaxiView surface movement video of JFK as a validation check of the algorithms.

In this paper, we ignore issues associated with aircraft type, where a gate is compatible with only a certain type of aircraft, and an aircraft occupying a gate may block its adjacent gates from being used. 


\section{Methods}

\section{A. Modeling of Taxi-in Process and Gate-occupancy Process}

Figure 2 shows a realization of the actual taxi-in process and gate-occupancy process.

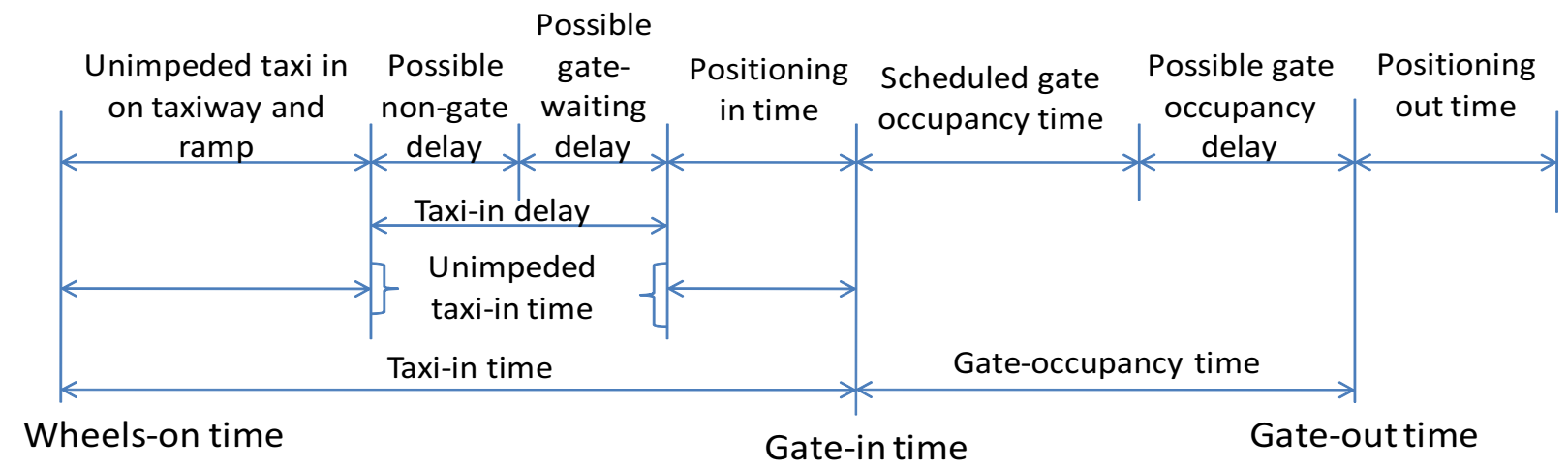

Figure 2. Taxi-in and gate-occupancy process

The taxi-in time includes the unimpeded taxi-in time and the taxi-in delays. These delays may be gate related or non-gate related (e.g., due to taxiway congestion). Note that the unimpeded taxi-in time (which is from wheels "on" to gate "in") includes the positioning-in time, the time to position an aircraft into the gate. The actual gate-occupancy time includes scheduled gate-occupancy time and possible gate-occupancy delay. We allow for the gate-occupancy delay to be negative in the case that a late arriving flight leaves on time and thus absorbs delay. ${ }^{27}$ A summary of the parameters and their sources in figure 2 are shown in table 3 .

Table 3. Required parameters and data sources

\begin{tabular}{l|l}
\hline \hline Parameter & Source \\
\hline Taxi-in time & Database field in BTS and ASPM \\
Unimpeded taxi-in time & Database field in ASPM estimated via a regression model ${ }^{25}$ \\
Gate-occupancy time & Pairing algorithm using BTS database \\
Positioning time & Deterministic value (estimate) \\
Gate-waiting delay & Calculated via equation $(2)$ (explained later) \\
\hline \hline
\end{tabular}

\section{B. Method 1: Estimation and Validation of Gate-waiting Delay}

This section describes several methods to infer gate-waiting delays based on information in the ASPM and BTS databases. We also describe a method to estimate gate-occupancy time by tracking the tail number of aircraft.

Gate-waiting delay is the difference between taxi-in delay and non-gate delay.

$$
\text { Gate-waiting delay }=\text { taxi-in delay }- \text { non-gate delay }
$$

However, no existing database contains the non-gate taxi-in delay, so it must be estimated. Based on interviews with United Airlines' airport managers, airport surface traffic is the main cause of non-gate delay, and this delay is usually smaller than 5 minutes. As a default, this paper assumes that up to 5 minutes of taxi-in delay is unrelated to gate delay; the remaining taxi-in delay is the estimation of gate-waiting delay. That is,

$$
\text { Gate-waiting delay } \approx \max (\text { Taxi-in delay }-5 \min , 0) .
$$

The maximum function ensures that the estimated delay is non-negative. For example, suppose that the unimpeded taxi-in time is 6 minutes. Then a 7 minute taxi-in time is estimated to contain 0 minutes of 
gate-waiting delay, while a 12 minute taxi-in time is estimated to contain 1 minute of gate-waiting delay. The method is partially justified by research indicating that gate delays are the dominant contributor to taxi-in delays. ${ }^{3,28}$ We have also checked the accuracy of equation 2 for a few sample flights from the surface operation video of JFK from Sensis Aerobahn TaxiView. From direct observation of the video, we identified the amount of time that an aircraft is moving (unimpeded taxi in), standing temporarily in front of a runway to cross or in front of another aircraft to move (taxi in delay due to traffic), and standing without any nearby runway or aircraft around it (gate-waiting delay). We observed the movements of two long taxi-in delayed flights and the estimation error was no more than 3 minutes. (One flight on 9/28/2008 was observed to have 32 minutes of gate-waiting delay compared with the original estimate of 34.4 minutes; Another flight on 10/10/2008 was observed to have 15 minutes gate-waiting delay compared with the original estimate of 16.4 minutes.)

Of course, this is an approximation, since the method assumes that non-gate-related delays comprise at most 5 minutes of the total taxi-in delay. We perform some sensitivity analysis on this 5 -minute threshold. The overall implication of the approximation is that the gate-delay statistic does not provide an exact magnitude, but can be useful as a relative metric to identify qualitative trends. For example, the identification of high-delay days appears to be insensitive to the exact value of the threshold (see figure 8 discussed later).

We now describe two specific methods for calculating gate-waiting delay from the ASPM and BTS databases and one method for calculating gate-occupancy time.

\section{Method 1a: ASPM Database}

The first method is based on data found in the ASPM database (Figure 3). Here, taxi-in delay is a specific field in the database (equal to the taxi-in time minus the unimpeded taxi-in time). The method extracts the taxi-in delay from the database and substitutes it into (2) to obtain the gate-waiting delay. A limitation of this method is that it does not track data associated with cancelled flights.

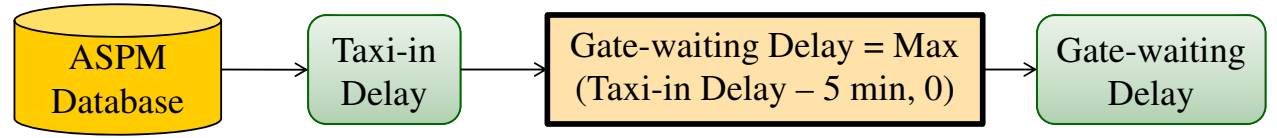

Figure 3. Method 1a: gate-waiting-delay estimation only using ASPM database

\section{Method 1b: ASPM and BTS Databases}

Figure 4 shows a modified version of the basic method that combines information from the ASPM and BTS databases. First, data on each flight is obtained from the BTS database (from the on-time performance table), including actual taxi-in time, date, carrier, and airport. The last three fields are used to look up the unimpeded taxi-in time from the ASPM database.

We assume that any date between June 1 and August 31 is mapped to the summer season for the purpose of looking up the unimpeded taxi-in time in the ASPM database. The taxi-in delay is then calculated as the difference between the actual taxi-in time and the unimpeded taxi-in time. The rest of the procedure is similar to method 1a.

Both methods $1 \mathrm{a}$ and $1 \mathrm{~b}$ have limitations in estimating gate-waiting delays. For example, to investigate the correlation between aircraft size and gate-waiting delay, method 1a is used because ASPM database has aircraft type information while BTS does not. On the other hand, when cancelations are considered, method $1 \mathrm{~b}$ is used since BTS database includes cancelations while ASPM does not. In gate-waiting-delay estimation in section V, "data source: ASPM database" refers to method 1a while "data source: BTS database" refers to method $1 \mathrm{~b}$. In this paper, method $1 \mathrm{a}$ is used in the carrier difference analysis and aircraft size correlation analysis. Method $1 \mathrm{~b}$ is used in other places.

\section{Method 2: Gate Occupancy Time Estimation Using Arrival-Departure Pairing}

Figure 5 shows a method to estimate the gate-occupancy time of a flight using its tail number. The aircraft tail number is usually available for a flight in the BTS database but not for a flight in the ASPM database. 


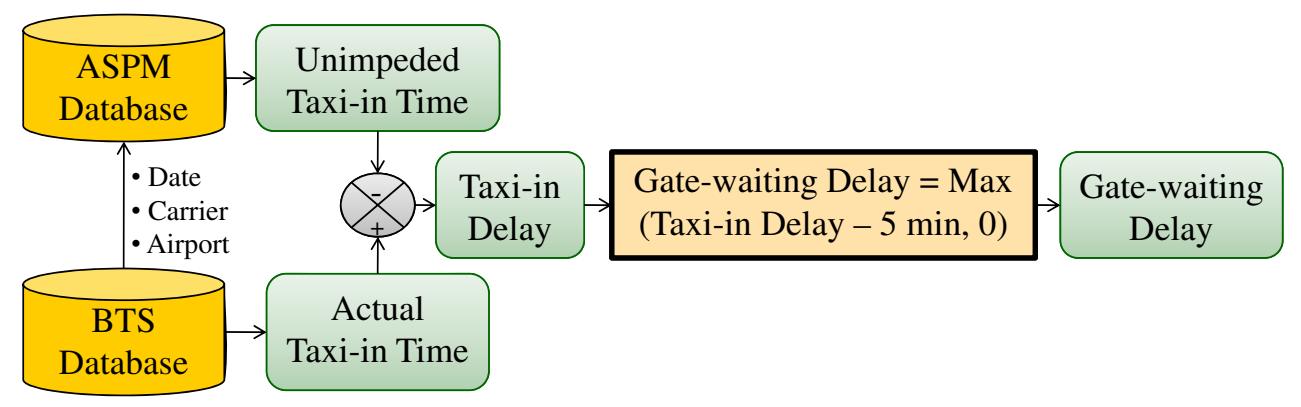

Figure 4. Method 1b: gate-waiting-delay estimation primarily using BTS database

Each arriving flight is paired with its departing flight as follows. For each arriving flight, the method searches for the earliest subsequent departure with the same tail number. If no such departure exists, or if the next available departure is more than 24 hours later, then the arriving flight is termed "unpaired" and is eliminated from the set. If a match is found, the gate occupancy time is the "out" time of the departing flight minus the "in" time of the arriving flight.

There are several limitations of this method. First, because of missing tail numbers, not all of the arrivals can be paired, resulting in missing turnaround times. For example, at ATL on 6/5/2007, 2.5\% of the flights are unpaired. Secondly, a missing departure can result in more than one arriving flight being paired with the same departing flight, resulting in an abnormally long gate-occupancy time for the earlier arrival. Finally, the method is limited to flights in the BTS database, so international flights are excluded.
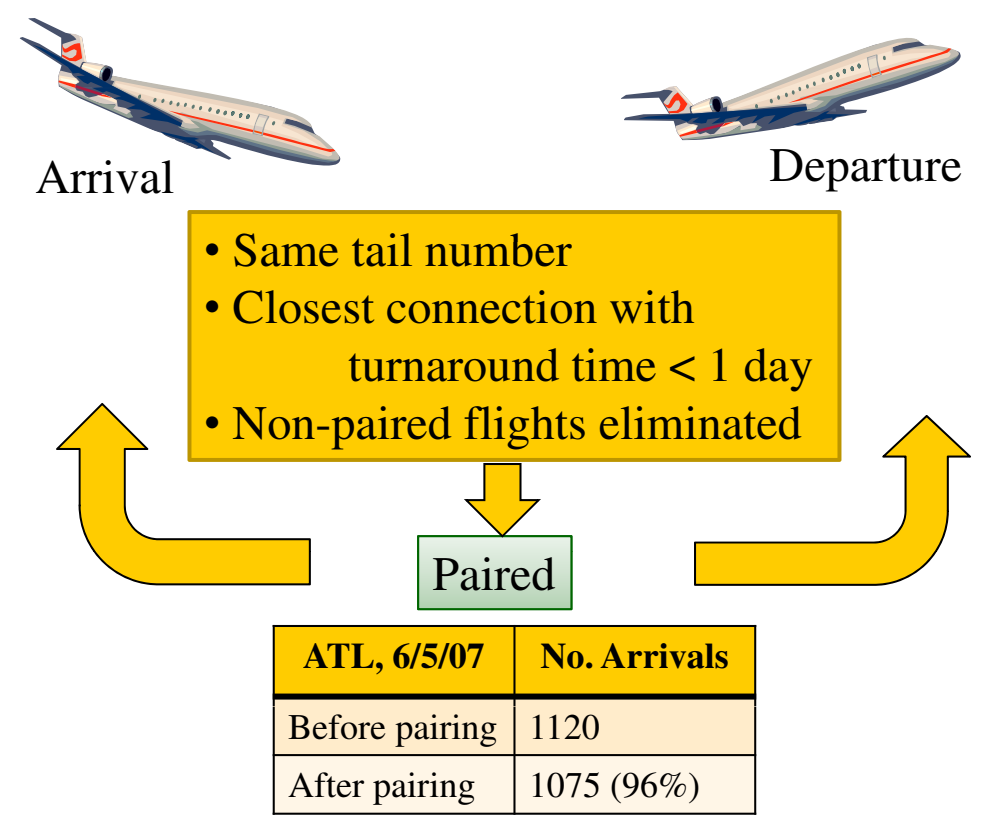

Figure 5. Method 2: arrival-departure pairing

\section{Results}

The results in this paper are based on data collected from the summer of 2007, June 1 through August 31 (92 days). 


\section{A. Overview of OEP 35 Airports}

We first give an overview of gate-waiting severity at the major US OEP 35 airports. More than 70 percent of passengers move through these airports. Delays at the OEP 35 airports have a ripple effect to other locations. Key FAA performance measures are based on data from these airports. ${ }^{29}$

\section{Airport Dimension}

Figure 6 shows the gate-waiting severity at the OEP 35 airports, calculated using Method 1b. The blue bars in the graph, show the number of days during which $30 \%$ or more of the flights experienced a strictly positive gate waiting. Out of the OEP 35 airports, 10 had at least 1 such day; the other 25 airports did not have any such days, so they are not shown on the $x$-axis. The red bars in the graph show the number of days during which $30 \%$ or more of the flights experienced a strictly positive gate-waiting delay and pulled into the gate after the scheduled arrival time. So if a flight a experienced gate-waiting delay due to early runway arrival, but ended up arriving at the gate on time, it is not counted. DFW, ATL and JFK have the most days during which $30 \%$ or more of the flights experienced a strictly positive gate waiting. But most of these days in the three airports were due to early arrivals, representing padding in the schedule and inflexibility/inability to change gate assignments when early arrivals happen.

Naturally, the estimate of gate delay is sensitive to the non-gate delay threshold from equation 2 . Table 4 gives a sensitivity analysis of the airport gate-delay ranking. The sensitivity analysis varies the non-gate delay threshold as well as the fraction threshold for identifying days with a high percentage of gate-delayed flights. Several airports appear near the top of most lists regardless of the values of these two parameters. (In this table, gate-waiting delays that arise from early arrivals are not subtracted out.)

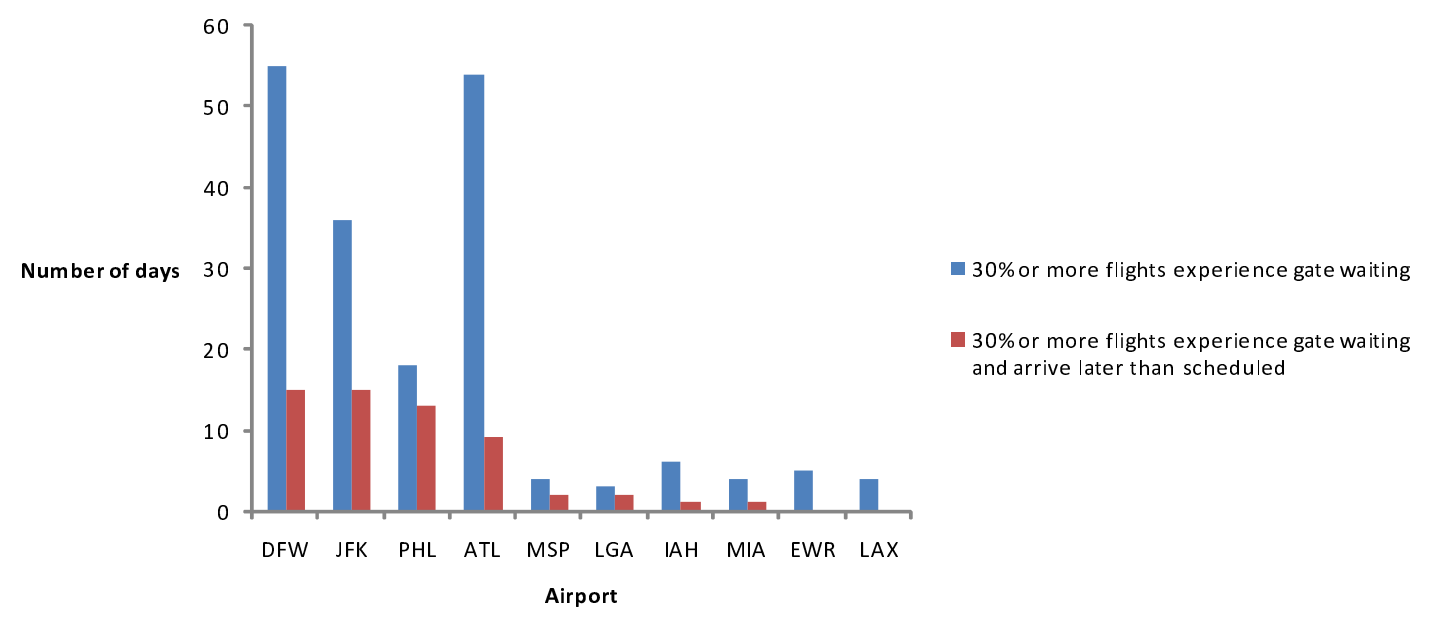

Figure 6. OEP 35 airport gate delays, method 1b

Figure 7 shows a gate-runway capacity ratio at some major US airports. This ratio is based on a dynamic apron capacity calculation. ${ }^{30}$ The calculation assumes that airports are busy non-hub airports and that all flights have a gate-service time of 60 minutes. This is only a rough estimation and does not consider the gateby-gate configuration of carrier and aircraft size. Based on the estimation, HNL is more likely to experience gate waiting delay than JFK when runway capacity is saturated. This does not mean that HNL has more gate delay, but rather if runway capacity were saturated, it would have more gate delay. JFK has a high gate/runway capacity ratio and also a high delay. The reason is that gate allocation is not proportional to flight volume such that airlines that have larger market share than gate allocation share contribute to high gate delays.

\section{Day Dimension}

Figure 8 shows the estimated daily total of gate-delay minutes at ORD for each day during the summer of 2007. The figure shows estimates using three different values for the non-gate delay threshold in equation 2. Naturally, the estimated amount of gate delay is smaller when the non-gate delay threshold is larger. The 
Table 4. Top 10 airports (if available) which have high gate-waiting fraction flights, method $1 \mathrm{~b}$

\begin{tabular}{c|c|c|c|c|c|c|c|c}
\hline \hline gate-waiting fraction threshold & $30 \%$ & $20 \%$ & $30 \%$ & $20 \%$ & $10 \%$ & $30 \%$ & $20 \%$ & $10 \%$ \\
\hline non-gate delay threshold (min) & 5 & 5 & 10 & 10 & 10 & 15 & 15 & 15 \\
\hline Top 10 airports where & DFW & DFW & PHL & JFK & JFK & PHL & JFK & JFK \\
fraction of flights & ATL & ATL & JFK & DFW & ATL & JFK & PHL & DFW \\
experienced gate waiting & JFK & JFK & ATL & PHL & DFW & & ATL & MIA \\
more than & PHL & LAX & & MIA & PHL & & DFW & PHL \\
the fraction threshold above & IAH & IAH & & ATL & LAX & & & ATL \\
(ordered by number of & EWR & DTW & & LGA & MIA & & & CLT \\
such "bad" days) & MIA & EWR & & & LGA & & & DCA \\
& MSP & PHL & & & EWR & & & EWR \\
& LAX & MSP & & & IAH & & & IAH \\
& LGA & ORD & & & DTW & & & LGA \\
\hline \hline
\end{tabular}

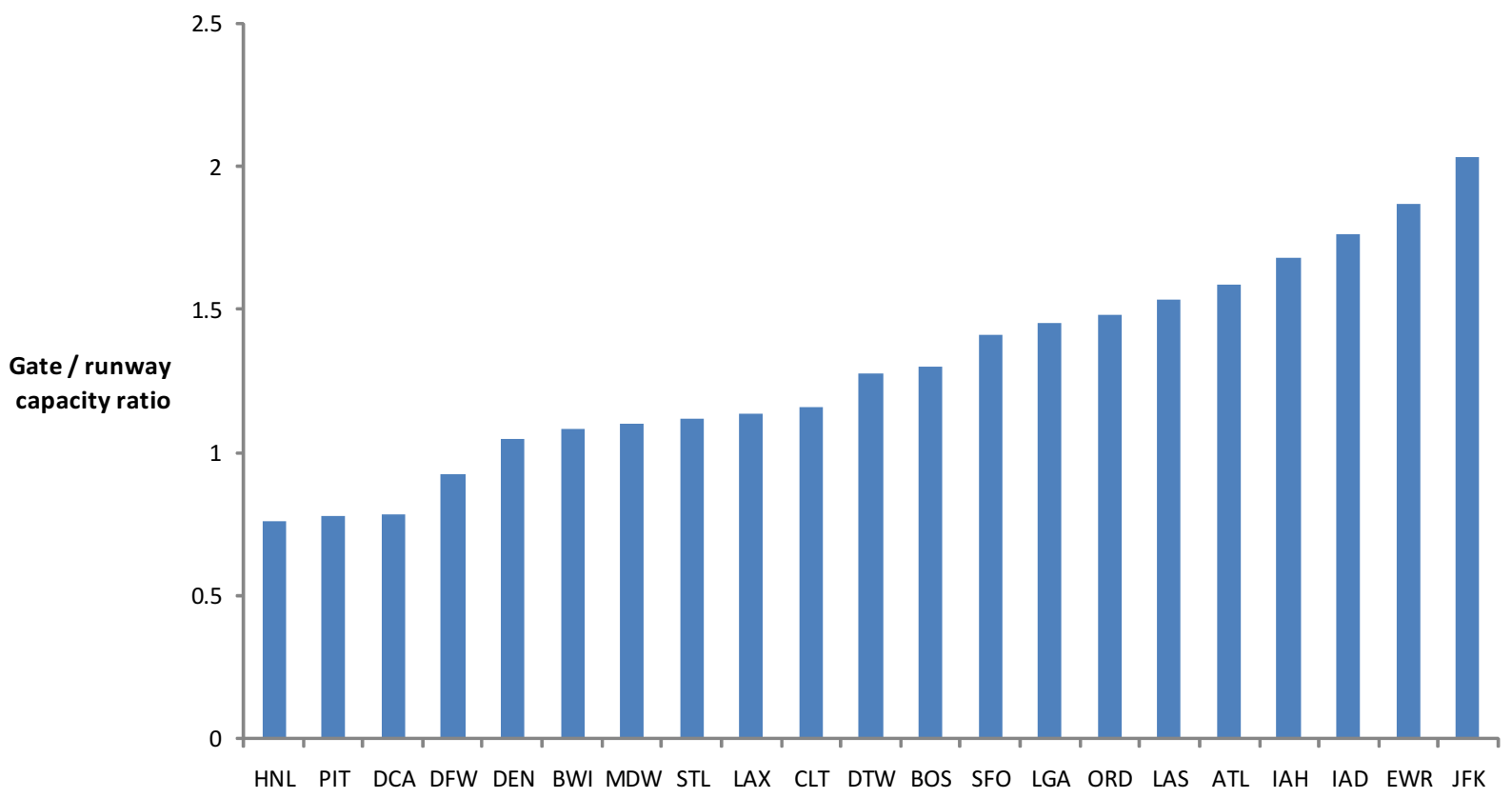

Figure 7. Rough gate/runway capacity ratio at some major US airports 
key observation from this figure is that extreme gate-waiting delays occur on several bad days at ORD. On most other days, delays are not that large. This observation is insensitive to the choice of the non-gate delay threshold. In other words, the two worst days $(7 / 18$ and 8/6) stand out as much worse than the other days, regardless of the non-gate delay threshold value. Analogous figures for other major airports show similar results.

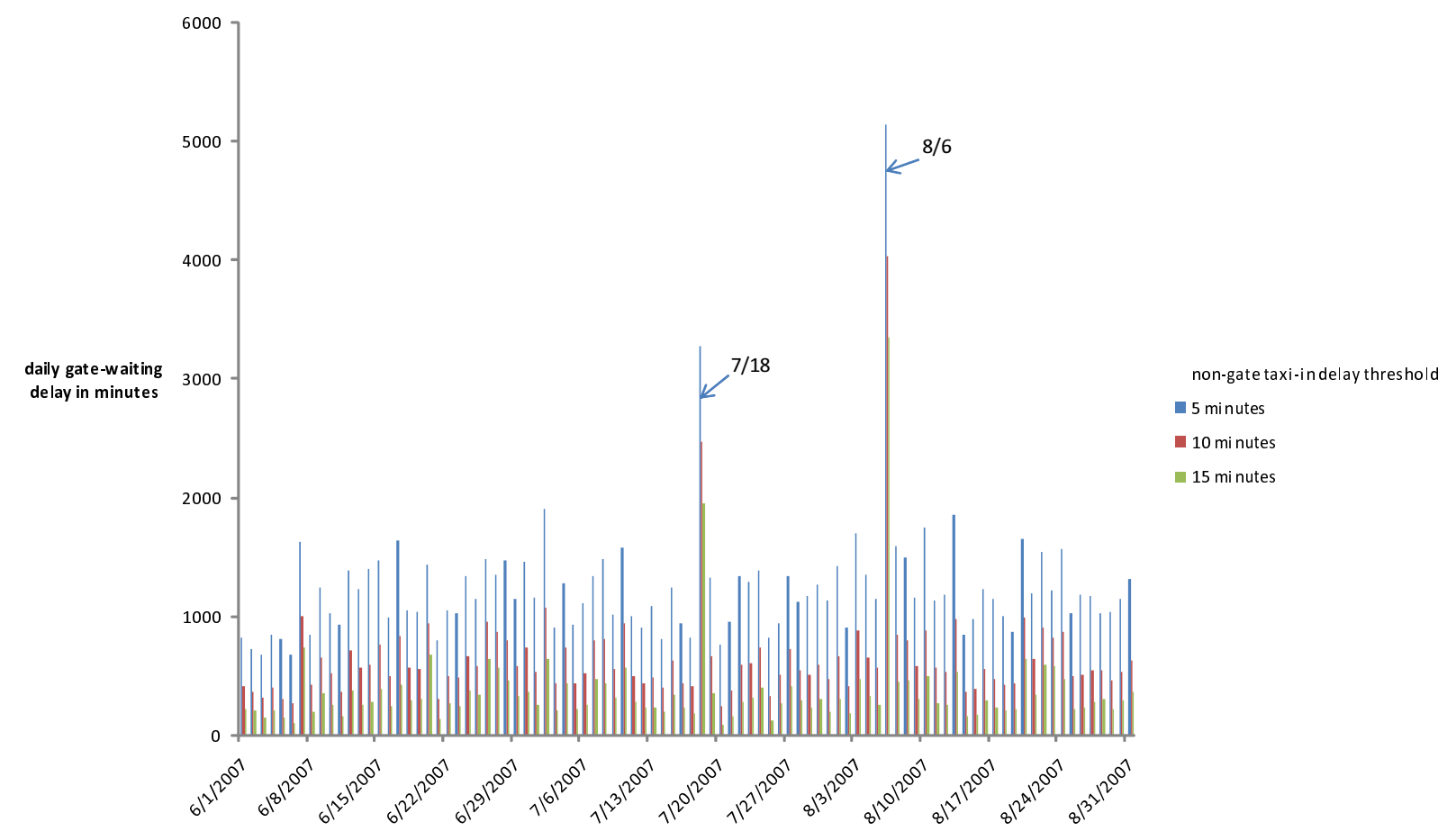

Figure 8. Gate-waiting delay by day at ORD, method $1 b$

\section{Time of Day Dimension}

Gate waiting is related to flight volume while flight volume is time dependent. As expected, gate-waiting percentage is also time dependent, as shown in figure 9. Furthermore, if a gate-waiting percentage of $30 \%$ is considered severe, then 8am to 10am at ATL and JFK, 11am to 9pm at DFW, and selected hours between 3pm to midnight at ATL, JFK, EWR, LAX are problematic time periods.

\section{Carrier Dimension}

Most gates in the US are managed by carriers exclusively, compared with central control by airports in Europe and Asia. Therefore it is natural to assume that gate-waiting delays are different among carriers since carriers have their own gate capacity and carriers determine the appropriate percentage of gate capacity to schedule flights. Usually, gate capacity has some slack because of spare gates. Flights can be accommodated by regular gates in the planning phase and spare gates in case of unexpected events, such as mechanical failures, schedule disruptions, etc. ${ }^{9}$

Based on analysis of the ASPM database, gate-waiting delays between carriers are often significantly different. Table 5 shows the average gate-waiting delays (per flight) for major carriers at several airports. For example, at JFK the three major carriers are JetBlue Airways (JBU), Delta Air Lines (DAL) with its subsidiary Comair (COM), and American Airlines (AAL) with its partner American Eagle Airlines (EGF). Based on a $t$-test, the differences in gate-waiting delays between any two pairs of carriers are found to be statistically significant (Table 6). Similarly, gate-waiting delay at other airports are usually significantly different between carriers if there are more than one major carriers. Another observation is that carriers with dominant market share in an airport tend to have higher gate-waiting delays, possibly due to their more aggressive scheduling based on the volume effect that aggregated uncertainties are less than the sum of 


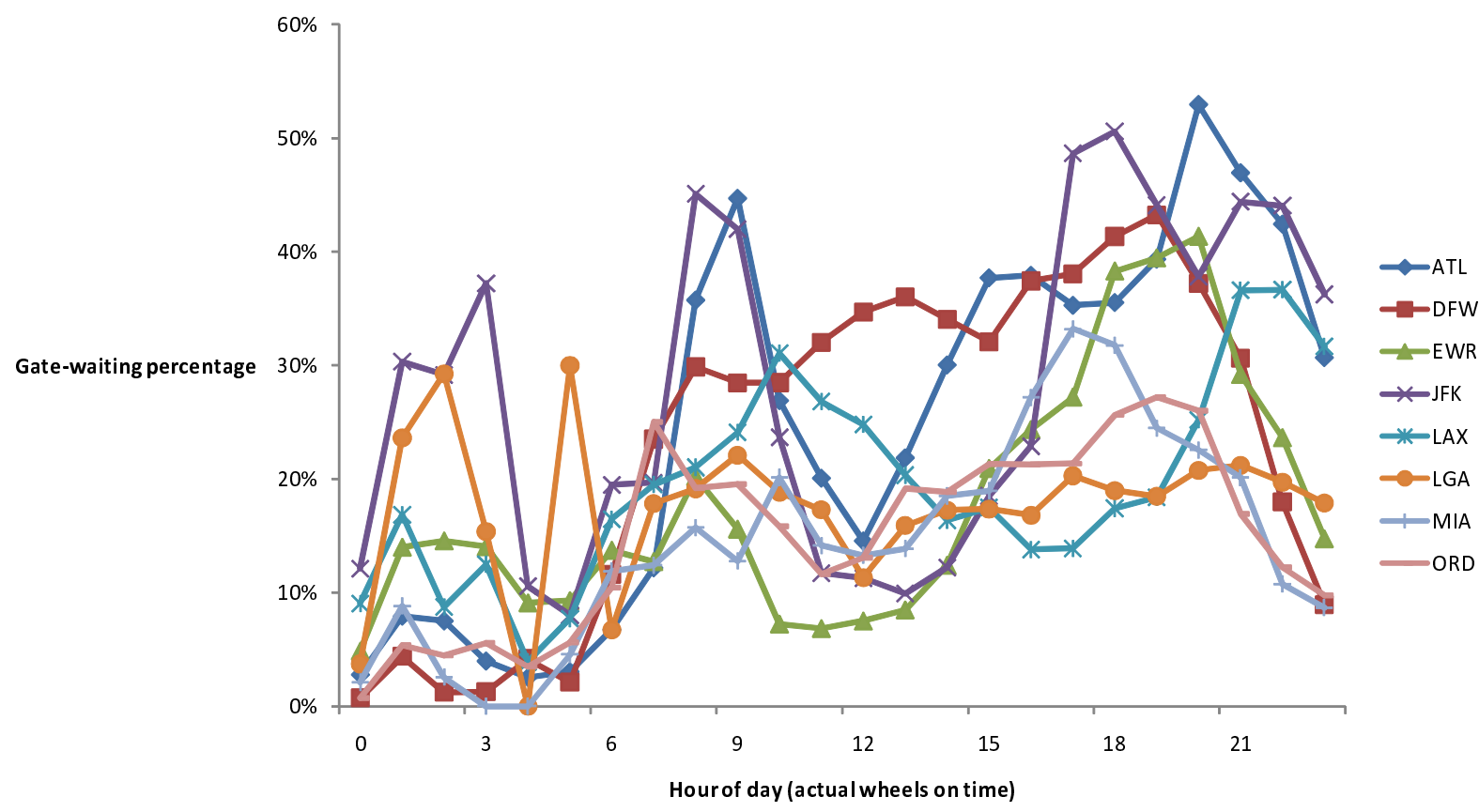

Figure 9. Gate-waiting percentage by time, method 1b (BTS data)

non-aggregated uncertainties. COA has a dominant market share at EWR and gate-waiting delays of COA flights are significantly higher than that of AAL. But AAL has a dominant market share at DFW, and its gate-waiting delay in DFW is higher than in EWR.

\section{Aircraft Size Dimension}

Figure 10 shows a scatter plot of gate-waiting delay versus aircraft size (in number of seats) for all flights at EWR during the summer of 2007. Table 7 gives the Pearson correlation coefficients between gate-waiting delay and aircraft size at several airports. In general, there is a relatively weak dependency of gate-waiting delay on aircraft size. Further, this correlation may be either positive or negative depending on the airports. For example, at EWR the correlation coefficient is .046, meaning that an aircraft with 100 more seats statistically experiences 4.6 more minutes of gate-waiting delay. At JFK, the correlation is negative meaning that larger aircraft tend to experience less delay. Either way, the conclusion is that large aircraft are not exempt from gate-waiting delays.

\section{B. High Gate-waiting Delay Days at ATL}

This section examines more closely the worst gate-waiting days occurring at one airport, ATL. In the summer of 2007, there were three extreme days during which delays were substantially worse than on other days. Figure 11 shows a break-down of the data by carrier. The figure shows the average gate-waiting percentage for the three major carriers at ATL on various days, as well as a summer average. For comparison, we also include a sample good day. The three major carriers are Delta Air Lines, Atlantic Southeast Airlines, and AirTran Airways, where Atlantic Southeast is a feeder carrier for Delta and also uses Delta's gates. Each of the three carriers has at least 200 arrivals per day while no others carriers at ATL have more than 35 arrivals per day. Although the figure shows that there are differences between the major carriers, no major carrier is exempt from gate-waiting delay on the "bad" days. That is, when it is bad for the airport, it is bad for all the carriers.

Figures 12 shows system characteristics on four days: June 5 (a sample good day), August 24 (the second-worst day), July 29 (the third-worst day), and June 11 (the worst day).

To explain the graphs, we compare the first two days. The first graph shows the percentage of arriving 
Table 5. Gate-waiting delay by major carriers at several major US airports, method 1a (ASPM data)

\begin{tabular}{|c|c|c|c|c|}
\hline Airport & Carrier & $\begin{array}{c}\text { Number of } \\
\text { arrivals }\end{array}$ & $\begin{array}{c}\text { Gate-waiting delay } \\
\text { mean (min) }\end{array}$ & $\begin{array}{c}\text { Gate-waiting delay } \\
\text { variance }(\mathrm{min})\end{array}$ \\
\hline \multirow[t]{2}{*}{ ATL } & $\mathrm{DAL}+\mathrm{ASQ}$ & 82185 & 2.42 & 41.03 \\
\hline & TRS & 23495 & 1.97 & 46.65 \\
\hline \multirow[t]{3}{*}{ JFK } & JBU & 16154 & 2.01 & 40.93 \\
\hline & $\mathrm{DAL}+\mathrm{COM}$ & 11891 & 6.39 & 226.75 \\
\hline & $\mathrm{AAL}+\mathrm{EGF}$ & 8140 & 2.46 & 47.44 \\
\hline \multirow[t]{4}{*}{ LGA } & $\mathrm{AAL}+\mathrm{EGF}$ & 10656 & 1.45 & 32.74 \\
\hline & $\mathrm{USA}+\mathrm{AWI}+\mathrm{PDT}$ & 10232 & 0.21 & 2.42 \\
\hline & $\mathrm{DAL}+\mathrm{COM}$ & 8406 & 1.71 & 32.24 \\
\hline & $\mathrm{COA}+\mathrm{CJC}$ & 4391 & 0.24 & 3.52 \\
\hline \multirow[t]{2}{*}{ EWR } & $\mathrm{COA}+\mathrm{BTA}$ & 37171 & 1.43 & 25.34 \\
\hline & AAL & 2052 & 0.77 & 7.21 \\
\hline DFW & AAL+EGF & 69951 & 2.65 & 52.78 \\
\hline \multirow[t]{7}{*}{ LAX } & SKW+ASQ & 13155 & 1.19 & 10.90 \\
\hline & $\mathrm{AAL}+\mathrm{EGF}$ & 13242 & 1.56 & 19.85 \\
\hline & SWA & 10568 & 0.69 & 7.36 \\
\hline & UAL & 9098 & 1.30 & 11.99 \\
\hline & $\mathrm{COA}+\mathrm{BTA}$ & 5870 & 2.62 & 35.70 \\
\hline & $\mathrm{ASA}+\mathrm{QXE}$ & 5135 & 0.42 & 6.69 \\
\hline & DAL & 4446 & 3.47 & 61.11 \\
\hline \multirow[t]{3}{*}{ MIA } & $\mathrm{AAL}+\mathrm{EGF}$ & 21903 & 2.25 & 71.06 \\
\hline & $\mathrm{GFT}+\mathrm{COA}$ & 3066 & 0.51 & 14.00 \\
\hline & $\mathrm{DAL}+\mathrm{COM}$ & 1209 & 0.25 & 7.72 \\
\hline
\end{tabular}

Table 6. Gate-waiting delay difference between major carriers at JFK, method 1a (ASPM data)

\begin{tabular}{l|rl}
\hline \hline Group & $t$ statistic & Difference \\
\hline JBU vs. (DAL+COM) & -29.8 & Significant \\
JBU vs. (AAL+EGF) & -19.56 & Significant \\
(DAL+COM) vs. (AAL+EGF) & 9.10 & Significant \\
\hline \hline
\end{tabular}




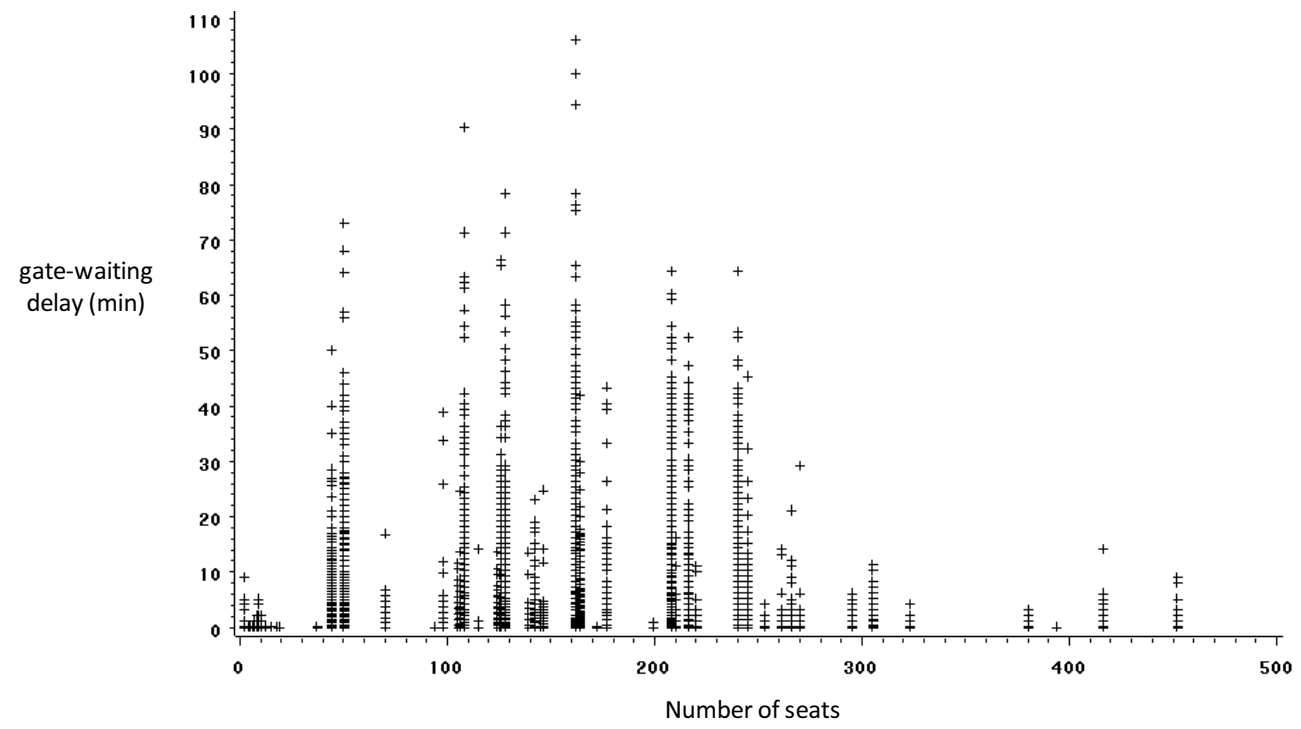

Figure 10. Scatter plot of gate-waiting delay in minutes by number of seats at EWR, method 1a (ASPM data)

Table 7. Pearson correlation coefficients between gate-waiting delay and aircraft size, ASPM data

\begin{tabular}{c|c}
\hline \hline & $\begin{array}{c}\text { Coefficients between } \\
\text { gate-waiting delay } \\
\text { and aircraft size }\end{array}$ \\
\hline ATL & -0.026 \\
JFK & -0.015 \\
LGA & 0.035 \\
EWR & 0.047 \\
DFW & 0.0089 \\
LAX & -0.019 \\
MIA & 0.068 \\
\hline \hline
\end{tabular}




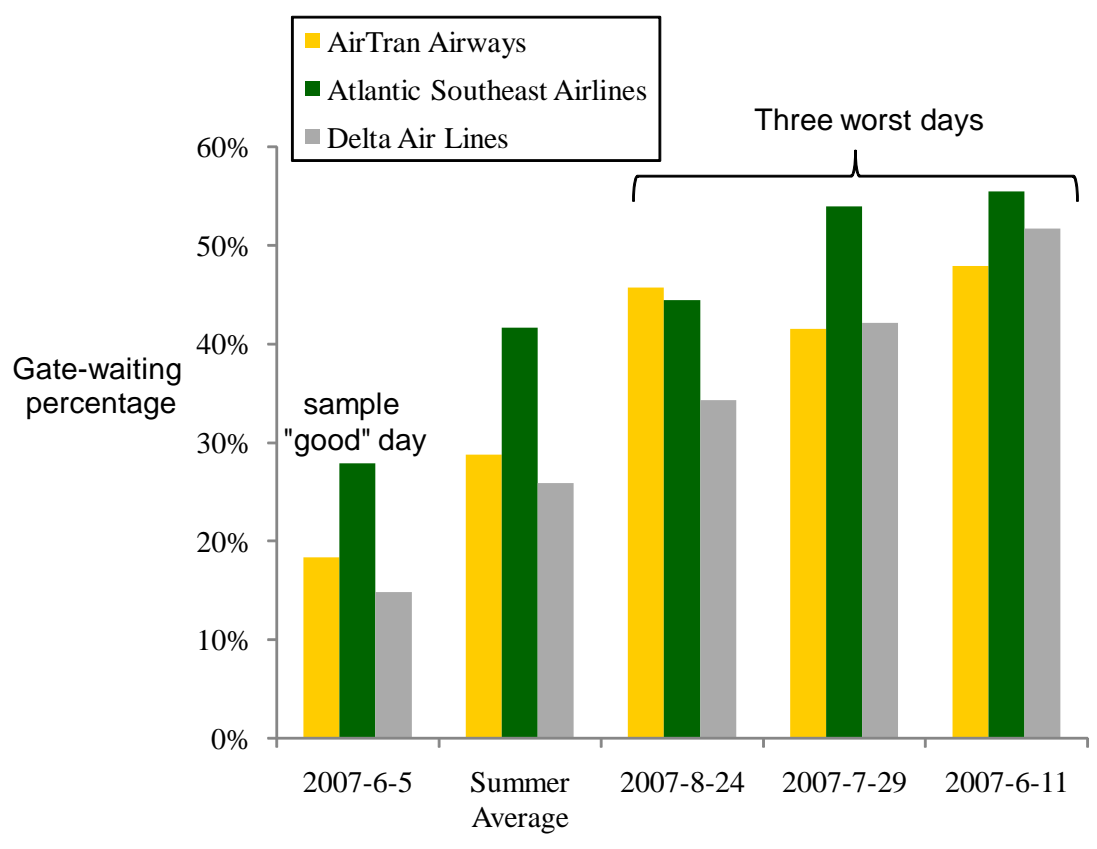

Figure 11. ATL gate-waiting delay by carrier on various days, method 1b(BTS data)

flights that experience a non-zero gate-waiting delay, grouped by hour of the day according to the actual wheels-on time of the arriving flight (method 1b). On the good day (June 5, 2007), the gate-waiting percentage is typically around $20 \%$ or less. There are three mild peaks in the morning, afternoon, and evening. In contrast, on the bad day (August 24, 2007), the gate-waiting percentage climbs steadily throughout the day to values near $100 \%$. The morning peak at 9am is still visible. In addition, a number of late arriving flights from the previous day contribute to a peak shortly after midnight.

The second graph shows a comparison of scheduled arrivals with actual arrivals (BTS data). (The scheduled arrivals correspond to arrivals at the gate while the actual arrivals correspond to arrivals at the runway.) On both days, the schedule has peaks roughly at $8 \mathrm{am}$ and $7 \mathrm{pm}$, with perhaps an intermediate peak around $4 \mathrm{pm}$. These peaks correspond with the three peaks in the gate-waiting percentage graph on the good day (June 5, 2007). The main difference between the two days is that on the good day, the actual operations roughly follow the scheduled operations; on the bad day, there is a significant schedule disruption at $7 \mathrm{pm}$, right at the evening peak. In particular, the arrival rate drops to near zero during this hour. The incoming flights are delayed and the peak is shifted later by about three hours.

The third graph shows the turnaround delay experienced by each aircraft, by time of day (method 2). Turnaround delay is defined as the actual turnaround time minus the scheduled turnaround time. (Based on this definition, it is possible for the turnaround delay to be negative. This typically occurs when an aircraft arrives late, but leaves on time. In particular, an aircraft that stays overnight, arriving late, but departing on-time the next morning can have a large negative turnaround delay.)

On the good day (June 5, 2007), turnaround delays are typically no more than \pm 60 minutes, though there are some aircraft that experience larger deviations from the schedule. On the bad day (August 24, 2007), the situation is somewhat different. First, there are significantly more aircraft with turnaround delays that exceed 60 minutes. Second, there is a gap in the arrival process around 7-8pm (consistent with the schedule disruption discussed previously). These effects are related.

Considering that both major carriers, Delta and AirTran, operate their flights in a hub and spoke network, this type of network relies heavily on predictability of its schedule so that crew and passengers can transfer efficiently between the arrival and departure banks. A disruption during this time can cause significant problems. For example, if half of the flights in the bank are delayed, then some of the flights that have arrived may wait for the delayed flights in order to establish continuity of passengers and crew. This causes 
June 5, 2007 (sample good day)
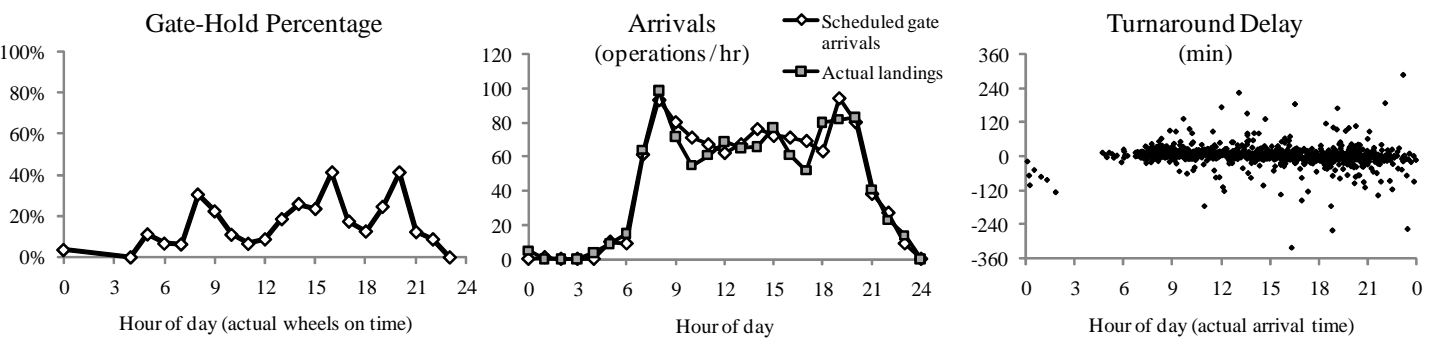

August 24, 2007 ( $2^{\text {nd }}$ worst day)
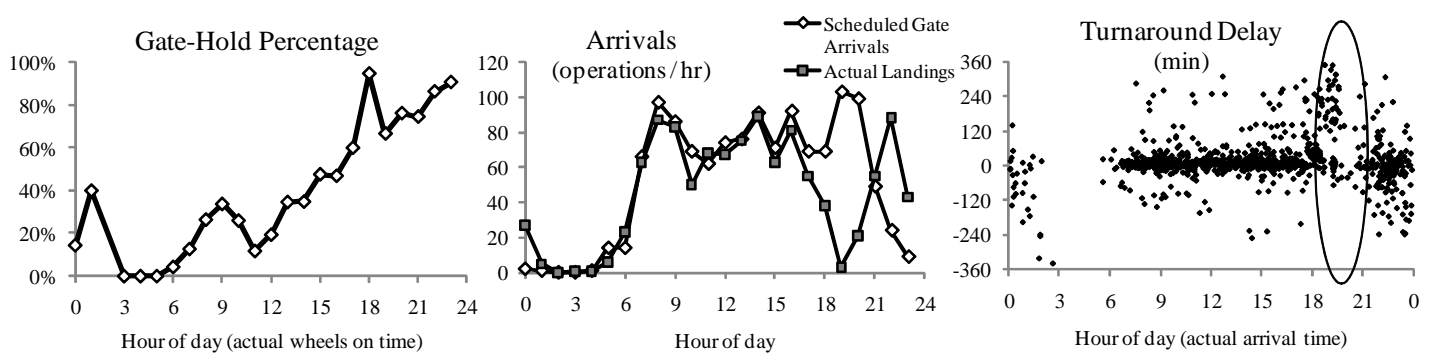

$\underline{\text { July } 29,2007 \text { ( } 3^{\text {rd }} \text { worst day) }}$
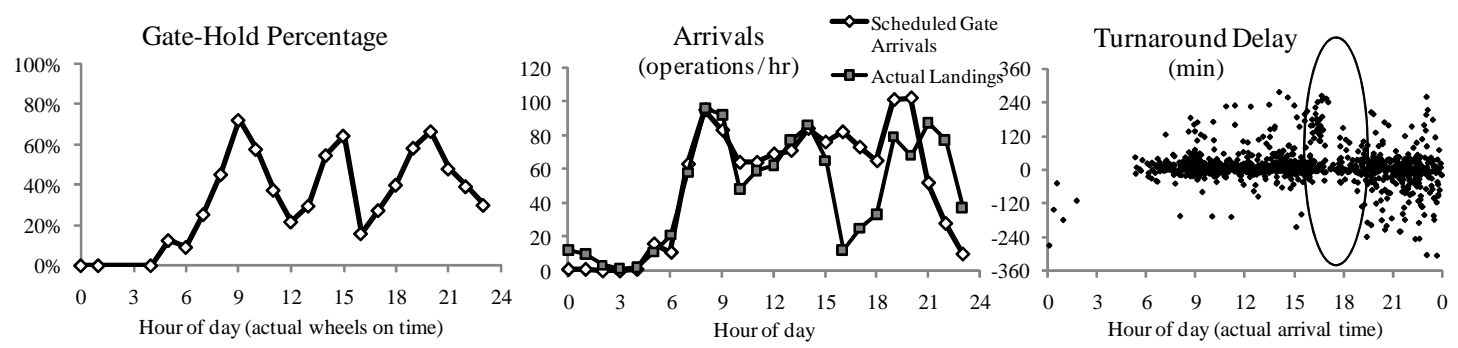

June 11, 2007 (worst day)
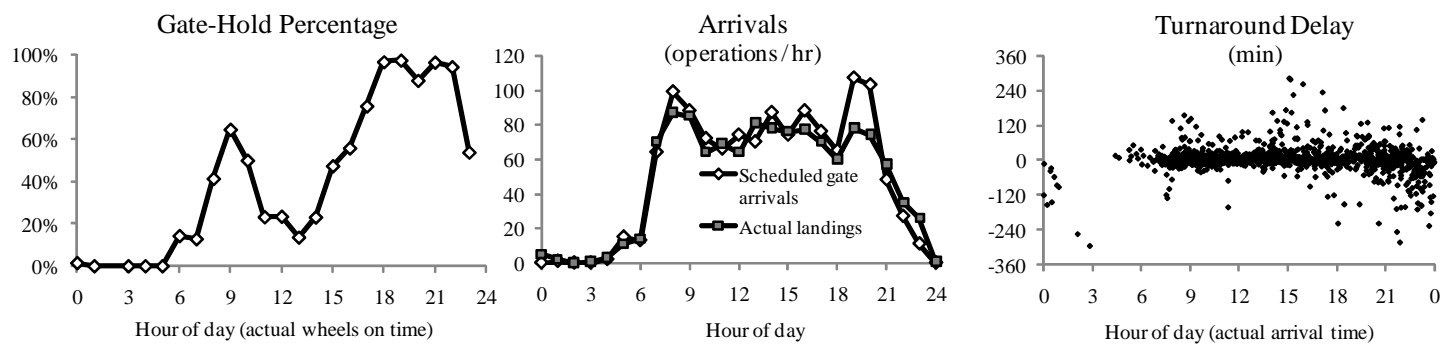

Figure 12. ATL gate-waiting delay on four example days, BTS data 
the aircraft to remain at their gates longer, thereby reducing the number of available gates and contributing to gate-waiting delay. Disruptions can also require crew changes which can delay aircraft at their gates.

In summary, the good day (June 5) is characterized by a close adherence to the schedule without much disruption. The bad day (August 24) is characterized by a significant schedule disruption, leading to long turnaround times. The long turnaround times limit gate availability and contribute to gate-waiting delays.

Now we consider the other two bad days at ATL: July 29 (the third-worst day) and June 11 (the worst day). From a qualitative perspective, July 29 is very similar to August 24. Specifically, there is a significant drop in arrival capacity in the late afternoon (this time around 4-6pm). This leads to significant deviations from the schedule where the disrupted arrival bank comes in several hours after its planned arrival time. This disruption leads to large increases in turnaround times, which in turn leads to large gate delays.

The system behavior on June 11 (the worst day), on the other hand, is qualitatively different than the other two bad days. In particular, June 11 does not appear to have a significant schedule disruption, except for perhaps a modest drop in arrivals around 7-8pm. One possible explanation is the number of cancellations (Figure 13, BTS data). This graph shows the number of arrival and departure cancellations by hour of the day. There is a large spike in departure cancellations around 5pm, followed by a large spike in arrival cancellations around 8pm. When departure flights are cancelled, the airplanes remain on the ground at the gate. Because the departure cancellations precede the arrival cancellations, there is a period of time when there are more airplanes on the ground, but the arrival rate into the airport has not yet been reduced, leading to a shortage of gates.

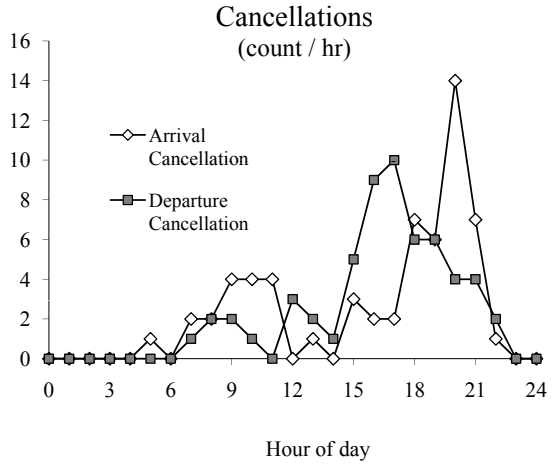

Figure 13. ATL cancellations on 6/11/2007, BTS data

In a similar manner, Figure 14 shows the hourly cancellation rate for the four focused days. The cancellation rate is low on June 5, 2007 when the gate-waiting performance is good. The cancellation rate is high on the three worst days, especially from 3-10pm, coinciding with the periods of high gate waiting. Cancellations can have a negative effect on gate delay, because a cancellation grounds an airplane for a period of time typically longer than it would otherwise remain at a gate, thus reducing overall gate capacity. In another words, flight cancellations reduce the number of active flights. This decreases the overall fleet's airborne time, which increases the overall fleet's ground time, which has the potential to increase gate delay.

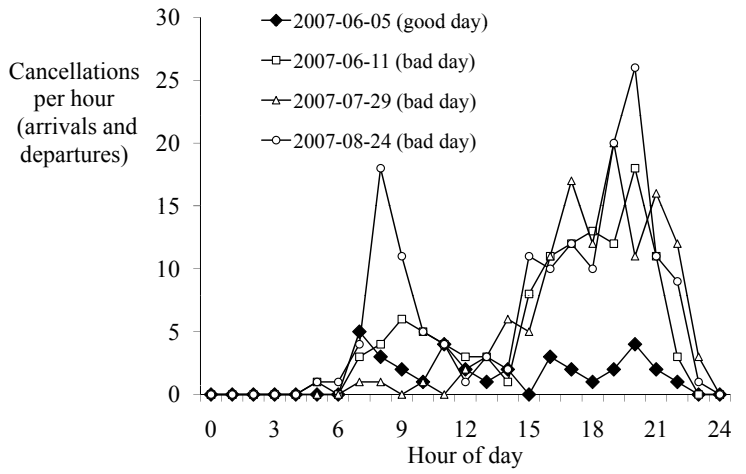

Figure 14. ATL cancellations on 4 focused days, BTS data

Moreover, departure cancellations often come before arrival cancellations, as in Figure 13. When a flight 
scheduled to depart late is cancelled, its aircraft may be reassigned to a later departure whose incoming flight is scheduled to arrive later and is also cancelled. This reduces flight delay by providing additional robustness in the schedule, but creates longer turnaround times which reduces the number of available gates.

Although the three worst gate-delay days have high cancellation rates, we have also observed many days with high cancellation rates and low gate-delay days. Thus, while high cancellation rates seem to occur on the worst days, they do not necessarily imply high gate delays.

\section{Conclusion}

This paper developed several methods to estimate gate-waiting delay using data from the ASPM and BTS databases. We applied the methods to the OEP 35 airports using data from the summer of 2007.

At airports where gate delay is high, there were statistically significant differences between the gate delays experienced by the major carriers, meaning that different carriers usually use a different portion of gate capacity for scheduling. For example, Delta schedules more aggressively on their gate capacity (overscheduling) than JetBlue at JFK. This suggests that more common gates could improve gate-waiting delay. But on days when there are major gate-waiting delays, large delays are experienced by all major carriers at some airports.

Aircraft type was correlated with gate-waiting delay. Generally, the observed correlation was small. Sometimes the correlation was negative, meaning that larger aircraft experienced slightly less gate delay, on average, such as in ATL and JFK. But larger aircraft do not experience lower average delays at all airports. On days when there is a major gate waiting, large delays were experienced by all major aircraft types at ATL.

Major daily gate-waiting delays are rare events (e.g., once a month at ATL). For most airports on most days, gate waiting was not an excessive problem. However, the gate-waiting delays were typically very bad on a few isolated days at each airport. We examined hour-by-hour statistics for the worst days at ATL and compared them with a typical "good" day. One common behavior of the worst days was the disruption of the schedule, including increased cancelations and longer turnaround times, resulting in more arriving flights than open gates.

Overall, functional origins of gate-waiting delay included compressed arrivals, extended gate occupancy times, reduced number of gates, and inflexible queueing disciplines (across carriers and within one carrier). Many of these origins are related to schedule disruptions which are the main common factor identified in the worst days. Other issues are strategic problems, such as the common-gate issue. Future work will study whether the unexpected events found in this paper can be taken into account in the decision making process and used to mitigate delays.

\section{Acknowledgments}

This research was partly funded by grants from the NASA Aeronautics Program, the FAA, and the internal Center for Air Transportation Systems Research Foundation. The authors appreciate technical contributions from Tony Diana, Akira Kondo, Stephanie Chung, Midori Tanino (FAA), Maria Consiglio, Brian Baxeley, Kurt Nietzche (NASA), Daniel DeLaurentis (Purdue), Chris Smith, Ben Levy, Bill Nix, Jeff Legge, George Hunter (Sensis), Norm Fujisaki, Terry Thompson, Mark Klopfenstein (Metron Aviation), Ed Stevens, Mary-Ellen Miller (Raytheon), Guillermo Calderon-Mesa, Maricel Medina Mora, Vivek Kumar, John Ferguson, Abdul Kara, George Donohue, Alexander Brodsky, Karla Hoffman, and Rajesh Ganesan (CATSR/GMU).

\section{References}

${ }^{1}$ FAA, "OEP Frequently Asked Questions," http://www.faa.gov/, 2007.

${ }^{2}$ Gross, D., Shortle, J. F., Thompson, J. M., and Harris, C. M., Fundamentals of Queueing Theory, John Wiley \& Sons, Inc, 4th ed., 2008.

${ }^{3}$ Idris, H. R., Anagnostakis, I., Delcaire, B., Hansman, R. J., Clarke, J.-P., Feron, E., and Odoni, A. R., "Observations of Departure Processes At Logan Airport to Support the Development of Departure Planning Tools," Air Traffic Control Quarterly, Vol. 7, 1999, pp. 229-257.

${ }^{4}$ Wang, D., Methods For Analysis Of Passenger Trip Performance In A Complex Networked Transportation System, Ph.D. thesis, George Mason Unversity, 2007. 
${ }^{5}$ Ramos, R. T., “AirTran doesn't control new gates, airport says," Atlanta Business Chronicle, February 2007.

${ }^{6}$ Idris, H. R., Observation and analysis of departure operations at Boston Logan International Airport, Ph.D. thesis, Massachusetts Institute of Technology, 2001.

${ }^{7}$ Horonjeff, R. and McKelvey, F., Planning and Design of Airports, McGraw-Hill Professional, 1993.

${ }^{8}$ Richards, J., "Self-Docking Saves Millions, Reduces Gate Delays at DFW," http://www.airportimprovement.com, September-October 2008.

${ }^{9}$ Bazargan, M., Airline Operations And Scheduling, Ashgate Pub Ltd, 2004.

${ }^{10}$ Bolat, A., "Procedures for providing robust gate assignments for arriving aircrafts," European Journal of Operational Research, Vol. 120, 2000, pp. 63-80.

${ }^{11}$ Haghani, A. and Chen, M.-C., "Optimizing gate assignments at airport terminals," Transportation Research Part A: Policy and Practice, Vol. 32, 1998, pp. 437-454.

${ }^{12}$ Yan, S. and Huo, C.-M., "Optimization of multiple objective gate assignments," Transportation Research Part A: Policy and Practice, Vol. 35, 2001, pp. 413-432.

${ }^{13}$ Bolat, A., "Assigning Arriving Flights at an Airport to the Available Gates," The Journal of the Operational Research Society, Vol. 50, 1999, pp. 23-34.

${ }^{14}$ Yan, S. and Chang, C.-M., "A network model for gate assignment," Journal of Advanced Transportation, Vol. 32, 1998, pp. 176-189.

${ }^{15}$ Yan, S. and Tang, C.-H., "A heuristic approach for airport gate assignments for stochastic flight delays," European Journal of Operational Research, Vol. 180, 2007, pp. 547567.

${ }^{16} \mathrm{Gu}$, Y. and Chung, C. A., "Genetic Algorithm Approach to Aircraft Gate Reassignment Problem," Journal of Transportation Engineering, Vol. 125, 1999, pp. 384-389.

${ }^{17}$ Yan, S., Shieh, C.-Y., and Chen, M., "A simulation framework for evaluating airport gate assignments," Transportation Research Part A: Policy and Practice, Vol. 36, 2002, pp. 885-898.

${ }^{18}$ Dorndorf, U., Jaehn, F., Lin, C., Ma, H., and Pesch, E., "Disruption management in flight gate scheduling," Statistica Neerlandica, Vol. 61, No. 1, 2007, pp. 92-114.

${ }^{19}$ Gillen, D. and Lall, A., "Developing measures of airport productivity and performance: an application of data envelopment analysis," Transportation research. Part E, Logistics and transportation review, Vol. 33, 1997, pp. 261-274.

${ }^{20}$ Andersson, K., Carr, F., Feron, E., and Hall, W. D., "Analysis and Modeling of Ground Operations at Hub Airports," 3rd USA/Europe Air Traffic Management R6BD Seminar, Napoli, 13-16 June 2000, 2000.

${ }^{21}$ Roling, P. C. and Visser, H. G., "Optimal Airport Surface Traffic Planning Using Mixed-Integer Linear Programming," International Journal of Aerospace Engineering, Vol. 2008, 2007.

${ }^{22}$ Fricke, H. and Schultz, M., "Improving Aircraft Turn Around Reliability," Third International Conference on Research in Air Transportation, 2008.

${ }^{23}$ BTS, "Airline On-Time Performance and Causes of Flight Delays," 2003.

${ }^{24}$ USpatent, "System and method of providing OOOI times of an aircraft," United States Patent 6154636, 112000.

${ }^{25}$ Kondo, A., The Derivation of the Unimpeded Taxi-out and Taxi-in Times in the Estimation of the Taxi Time Delays, Office of Aviation Policy, Federal Aviation Administration, technical note 2009 no. 1 ed., 2009.

${ }^{26}$ ASPM, F., "data reference guide," 2008.

${ }^{27} \mathrm{Xu}$, N., Method for Deriving Multi-Factor Models for Predicting Airport Delays, Ph.D. thesis, George Mason University, 2007.

${ }^{28}$ Gosling, G. D., "Development of a framework for assessing the benefits of airportsurface traffic automation," Control Systems Technology, IEEE Transactions on, Vol. 1, 1993, pp. 155-167.

${ }^{29}$ FAA, "OEP Frequently Asked Questions - OEP 35 Airports," July 2007.

${ }^{30}$ de Neufville, R. and Odoni, A., Airport Systems: Planning, Design, and Management, McGraw-Hill Professional, 1st ed., October 2003

\section{Author Biographies}

Jianfeng Wang is a doctoral candidate and research assistant in the Center for Air Transportation Systems Research at George Mason University.

John F. Shortle is an Associate Professor of Systems Engineering and Operations Research at George Mason University (GMU). His research interests include simulation and queueing applications in air transportation and telecommunications. He is a member of the Center for Air Transportation Systems Research and the Center for Network-Based Systems. Previously, he worked in the telecommunications industry at US WEST Advanced Technologies. He received a Ph.D. and M.S. in industrial engineering and operations research at UC Berkeley in 1996.

Juan Wang is a graduate student and research assistant in the Center for Air Transportation Systems Research at George Mason University.

Lance Sherry is the Executive Director of the Center for Air Transportation Systems Research at George 
Mason University and an Associate Professor of Systems Engineering and Operations Research. He received his Ph.D. in industrial and management systems engineering in 1999 from Arizona State University. 